\title{
Wireless Communication with Chaos
}

\author{
Hai-Peng Ren, ${ }^{1,2}$ Murilo S. Baptista, ${ }^{2}$ and Celso Grebogi ${ }^{2,3}$ \\ ${ }^{1}$ Department of Information and Control Engineering, Xi'an University of Technology, Xi'an 710048, China \\ ${ }^{2}$ Institute for Complex System and Mathematical Biology, SUPA, University of Aberdeen, Aberdeen AB24 3UE, United Kingdom \\ ${ }^{3}$ Freiburg Institute for Advanced Studies, Freiburg University, 79104 Freiburg, Germany
}

(Received 5 December 2012; published 29 April 2013)

\begin{abstract}
The modern world fully relies on wireless communication. Because of intrinsic physical constraints of the wireless physical media (multipath, damping, and filtering), signals carrying information are strongly modified, preventing information from being transmitted with a high bit rate. We show that, though a chaotic signal is strongly modified by the wireless physical media, its Lyapunov exponents remain unaltered, suggesting that the information transmitted is not modified by the channel. For some particular chaotic signals, we have indeed proved that the dynamic description of both the transmitted and the received signals is identical and shown that the capacity of the chaos-based wireless channel is unaffected by the multipath propagation of the physical media. These physical properties of chaotic signals warrant an effective chaos-based wireless communication system.
\end{abstract}

DOI: 10.1103/PhysRevLett.110.184101

PACS numbers: 05.45.Vx

Nowadays, wireless communication [1,2], ranging from satellite communication and global positioning system (GPS) navigation [3], underwater wireless sensor networks [4], personal mobile phones to portable Wi-Fi applications, is closer to our daily experiences than it used to be. Reliability of the communication is compromised due to several physical constraints affecting the information signal, such as amplitude damping, filtering that causes modification both in the phase and amplitude of the signal, multipath propagation (a signal that travels along many different paths and arrives many times at the receiving location) that causes serious interference, time-varying characteristics, and noise.

Wireless communication usually has worse performance than wired channel communication. One of the main reasons is the multipath propagation, which is caused by a series of unavoidable factors, including atmospheric ducting, ionospheric reflection and refraction, and reflection from water bodies [5] and terrestrial objects such as mountains and buildings [6].

The main challenge in communication is to be able to realize what is being transmitted $\left[x_{T}(t)\right]$ from what is being received $\left[x_{R}(t)\right]$. A standard model for the wireless channel is given by a linear time-varying filter channel [7] $x_{R}(t)=$ $\sum_{j=1}^{L} a_{j}(t) x_{T}\left(t-\tau_{j}\right)+\eta(t)$, where $a_{j}(t)$ represents a timevarying attenuation that can be complex [8] in order to represent a change in the phase of the received signal (modeling the action of the filter), $\tau_{j}$ represents the time delay of the $j$ th path that the signal travels, and $\eta(t)$ represents additive noise.

The use of chaos in communication has attracted much attention [9-22] due to many intrinsic properties of chaos, such as sensitivity to initial conditions, broadband and orthogonality, and the fact that chaotic signals can be generated by low power, low cost, small area electronic circuits. If chaotic signals are transmitted over ideal channels, Ref. [9] has shown that the maximum rate at which information can be transmitted is given by the topological entropy of the chaotic system. The physical constraints on the nonideal chaos-based communication channel were modeled in terms of additive noise [12-14,17] and, recently, adding filtering effects [21,23-26]. As chaotic communication has been successfully used to achieve higher bit rate in a commercial wired fiber-optic channel [22], chaotic communication research in practical nonideal communication channels became a focus in research $[3,5,26,27]$. So far, however, there is no work that has dealt with the information capacity of the nonideal chaos-based wireless communication channel or that has described how chaos can be used to create a wireless communication system [28]. In particular, what should one expect about the fundamental dynamical properties of $x_{R}$ when $x_{T}$ is a chaotic wave signal?

In this work, we first show that if $\lambda_{\mathrm{T}}$ represents the spectra of Lyapunov exponents (LEs) of the transmitted chaotic signal $x_{T}$, then $\lambda_{\mathbf{T}} \in \lambda_{\mathbf{R}}$, where $\lambda_{\mathbf{R}}$ represents the spectra of LEs of the received signal $x_{R}(t)$. We also show that if a Poincaré mapping of the transmitting chaotic flow can be constructed by $x_{T}\left(t+n T_{s}\right)=F^{n T_{s}}\left(x_{T}(t)\right), T_{s}$ is the sampling time such that $x_{T}(n+1)=J x_{T}(n)$, and if the bandwidth of the filter is large enough [Eq. (4)], then

$$
x_{R}(n+1)=J x_{R}(n),
$$

where $J$ is a one-dimensional constant Jacobian that has one eigenvalue larger than 1 . We then evaluate the information capacity [see Eqs. (9) and (10)] of the chaos-based communication channel described by Eqs. (2) and (3), suggesting an effective chaos-based wireless communication system. The first fundamental finding shows an important property of chaotic signals. They preserve their LEs 
after being transmitted through wireless channels. The subsequent two results are a consequence of this finding. If $J$ is a hyperbolic 1D constant Jacobian that has one eigenvalue larger than 1 , the amount of information in the transmitted chaotic signal is equal to the positive Lyapunov exponent, i.e., $\lambda_{T 1}$ per iteration. The information in the received signal is also preserved, and equals $\lambda_{T 1}$ per iteration. Furthermore, if the condition in Eq. (1) is satisfied, then the decoding of the information can be trivially performed.

To model the chaos-based wireless channel (CBWC), we make the attenuation parameter $a_{j}$ in the linear timevarying filter channel real and apply the filtering action to the transmitted signal, yielding

$$
x_{R}(t)=\sum_{j=1}^{L} e^{-\beta \tau_{j}} w\left(t-\tau_{j}\right),
$$

$w(t)$ representing the transmitted signal $x_{T}(t)$ after being filtered by

$$
\dot{w}(t)=-\alpha_{1} w(t)+\alpha_{2} x_{T}(t),
$$

where the attenuation is modeled by an exponential decay with coefficient $-\beta$, and the time delay $\tau_{j}$ is determined by the length of the path $j$ connecting the transmitting and the receiving stations (divided by the wave speed). The attenuation in the free-space wireless channel results from the radiation inverse square law that describes the decay of the power of the electromagnetic wave as $\propto 1 / d^{2}$ ( $d$ is distance). In practice [31], due to the media or obstacles, the decay can be exponential for sufficiently long distances. Equations (2) and (3) show that the received signal $x_{R}(t)$ is a nontrivial function of $x_{T}(t)$; hence, especially because of multipath propagation, the decoding of information [9] from $x_{R}(t)$ becomes very difficult to perform [26] (see Supplemental Material [32] for the illustration).

Assume for now that the only constraint in the communication channel is the filtering in Eq. (3). As shown in Ref. [23], the effect of a linear filtering on a chaotic signal is the addition of one negative LE to the original signal. The positive Lyapunov exponents remain unchanged. According to the equation for the Lyapunov dimension $D_{L}=k+\frac{\sum_{i=1}^{k} \lambda_{i}}{\left|\lambda_{k+1}\right|}$, where $\lambda_{k+1}$ is the $(k+1)$ th largest Lyapunov exponent of the signal $x_{T}$ such that $\sum_{i=1}^{k} \lambda_{i}>0$ and $\sum_{i=1}^{k+1} \lambda_{i}<0$. From the linear system theory [33], the filter in Eq. (3) is stable if $\alpha_{1}>0$ and the Lyapunov exponent of the filter is $-\alpha_{1}$. So, if

$$
\left|\lambda_{k+1}\right|<\alpha_{1} \text {, }
$$

the Lyapunov dimension of the filtered signal is not altered and, consequently, as shown in Refs. [24,25], information can be transmitted in a physical media that linearly filters signals. Even if Eq. (4) is not satisfied, communication can be implemented with low bit error rate [25]. However, Eq. (4) is one condition that needs to be satisfied for our communication system to be feasible. In an experimental setup, $\alpha_{1}$ of the channel can be experimentally measured.

To study the combined effect of filtering, damping, and multipath, we now study the continuous time signal as given by Eqs. (2) and (3), with $\alpha_{1}>0$ and $\alpha_{2}>0$. We consider that $x_{T}$ is equal to the variable $z(t)$ of the Lorenz system [34] given by $\dot{x}(t)=a[y(t)-x(t)], \dot{y}(t)=c x(t)-$ $y(t)-x(t) z(t), \dot{z}(t)=x(t) y(t)-b z(t)$, where $a=16, b=$ 4 , and $c=45.92$.

Assuming that the channel has only two paths, we get the received signal $x_{R}(t)=e^{-\beta \tau_{1}} w\left(t-\tau_{1}\right)+$ $e^{-\beta \tau_{2}} w\left(t-\tau_{2}\right)$.

In order to rewrite $x_{R}(t)$, a time-delay expression, as the solution of an autonomous system of ordinary differential equations (ODEs), we define the variables as follows: $x_{1}(t)=w(t-\Delta t), \quad x_{i}(t)=x_{i-1}(t-\Delta t)=w(t-i \Delta t)$, for $1<i \leq k_{2}$ and $i \in \mathbb{Z}$. $\Delta t$ is a sufficiently small time interval, so that the delays $\tau_{1}$ and $\tau_{2}$ can be expressed by $\tau_{1}=k_{1} \Delta t$ and $\tau_{2}=k_{2} \Delta t$, and the derivative of the new variables can be written as $\dot{x}_{1}(t)=\left[w(t)-x_{1}(t)\right] / \Delta t$, $\dot{x}_{i}(t)=\left[x_{i-1}(t)-x_{i}(t)\right] / \Delta t$. Then, the received signal becomes $x_{R}(t)=e^{-\beta \tau_{1}} x_{k_{1}}(t)+e^{-\beta \tau_{2}} x_{k_{2}}(t)$. By defining new variables as $w_{1}(t)=e^{-\beta \tau_{1}} x_{k_{1}}(t)+e^{-\beta \tau_{2}} x_{k_{2}}(t), w_{i}(t)=$ $x_{k_{2}-i+1}(t), 2 \leq i \leq k_{2}, \quad w_{k_{2}+1}(t)=w(t), \quad w_{k_{2}+2}(t)=z(t)$, $w_{k_{2}+3}(t)=y(t)$, and $w_{k_{2}+4}(t)=x(t)$, we have the $\left(k_{2}+4\right)$ dimensional system given by

$$
\dot{\mathbf{S}}(t)=\mathbf{F}[\mathbf{S}(t)],
$$

where $\mathbf{S}=\left[w_{1}, w_{2}, \ldots, w_{k_{2}+4}\right]$ is the state variable vector and $x_{R}(t)=w_{1}(t)$ is the received signal. Here, $\dot{w}_{1}(t)=\left[-w_{1}(t)+e^{-\beta \tau_{2}} w_{2}(t)+e^{-\beta \tau_{1}} w_{k_{2}-k_{1}+2}(t)\right] / \Delta t$. The Jacobian matrix of the system (5) is written as

$$
\mathbf{J}_{c}=\left[\begin{array}{ll}
J_{c 11} & J_{c 12} \\
J_{c 21} & J_{c 22}
\end{array}\right]
$$

where

$$
\begin{aligned}
& J_{c 22}=\left[\begin{array}{cccc}
-\alpha_{1} & \alpha_{2} & 0 & 0 \\
0 & -b & w_{k_{2}+4} & w_{k_{2}+3} \\
0 & w_{k_{2}+4} & -1 & c-w_{k_{2}+2} \\
0 & 0 & a & -a
\end{array}\right], \quad J_{c 21}=\mathbf{0} \text {, } \\
& J_{c 12}=\left[\begin{array}{cc}
\mathbf{0} & \mathbf{0} \\
1 / \Delta t & \mathbf{0}
\end{array}\right] \text {, } \\
& J_{c 11}=\left[\begin{array}{ccccc}
-1 / \Delta t & e^{-\beta \tau_{2} / \Delta t} & \mathbf{0} & e^{-\beta \tau_{1}} / \Delta t & \mathbf{0} \\
& -1 / \Delta t & 1 / \Delta t & \mathbf{0} & \mathbf{0} \\
& & \ddots & \ddots & \\
& & & -1 / \Delta t & 1 / \Delta t \\
& & & & -1 / \Delta t
\end{array}\right] \text {. }
\end{aligned}
$$

The matrix (6) is composed of the $\left(k_{2}\right)$-dimensional left-upper upper-triangular submatrix $J_{c 11}$ and the 
four-dimensional right-down submatrix $J_{c 22}$, which is the Jacobian matrix of the original Lorenz system and the filter. $J_{c 21}$ and $J_{c 22}$ do not contribute to the LEs. As a consequence, the Lyapunov exponent spectrum of system (5) is given by

$$
\left.\lambda_{\mathbf{R}}=[\underbrace{-1 / \Delta t, \ldots,-1 / \Delta t}_{k_{2}},-\alpha_{1}, \lambda_{3}, \lambda_{2}, \lambda_{1})\right],
$$

where $-\alpha_{1}$ is the Lyapunov exponent of the filter, and $\lambda_{3}$, $\lambda_{2}, \lambda_{1}$ are the LEs of the original Lorenz system. Requiring $\Delta t \rightarrow 0$ results in a number of $k_{2}$ LEs that are equal to $-\infty$, which are produced by the delays of the paths. Numerically, we consider $\Delta t$ to be sufficiently small such that $-1 / \Delta t \ll-\alpha_{1}$. The numerical calculation of the LEs of the system in Eq. (5) with $\tau_{1}=0.2, \tau_{2}=0.5, \Delta t=$ $0.02, \alpha_{1}=-30, \alpha_{2}=30$ produces a LE spectra that contains the three LEs of the original Lorenz system. It should be noted that we can define the variables as aforementioned for more than two path propagations so that a similar Jacobian can be obtained and, therefore, only negative LEs $(-1 / \Delta t)$ are generated from the multipath propagations. In conclusion, as claimed earlier, the LEs are preserved; i.e., the received signal contains the LEs of the transmitted signal [35,36]. Equivalent results for maps can be found in the Supplemental Material [32].

Because the LEs are preserved, if the communication uses a chaotic signal produced by a piecewise linear system with a constant Jacobian, the received signal also possesses a piecewise linear description producing the same LEs. This is correct if the flow can be transformed by a Poincare map into a piecewise linear system with a constant Jacobian. Corron et al. [38] designed a circuit that has this property. It is described by

$$
\ddot{x}-2 \xi \dot{x}+\left(\omega^{2}+\xi^{2}\right)(x-s)=0,
$$

where $0<\xi \leq \ln (2), \omega=2 \pi$ are parameters, the variable

$$
s=\operatorname{sgn}(x)= \begin{cases}1 & x \geq 0 \\ -1 & x<0\end{cases}
$$

only switches its value at the time when $\dot{x}=0$, keeping its value at any other times. System (7) is a hybrid system by combining the continuous state $x$ with the discrete switched signal $s$. If $\xi=\ln 2$, this system is chaotic with one positive Lyapunov exponent equal to $\ln 2$. The time delay embedding three-dimensional phase space of system (7) is shown in Fig. 1(a), which is rather similar to the Matsumoto-Chua circuit [39]. By sampling the state variable $x(t)$ with a sampling time of $T_{s}=1$, we obtain a time Poincaré mapping that produces the return map shown in Fig. 1(b), analytically described by

$$
x_{n+1}= \begin{cases}2 x_{n}-1 & x_{n} \geq 0 \\ 2 x_{n}+1 & x_{n}<0 .\end{cases}
$$

If $x(t)$ is sent over a wireless channel, i.e., $x_{T}(t)=x(t)$ in Eq. (3), with two propagation paths, damping rate equal to (a)

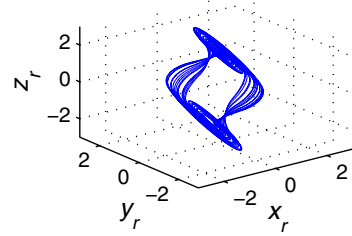

(c)

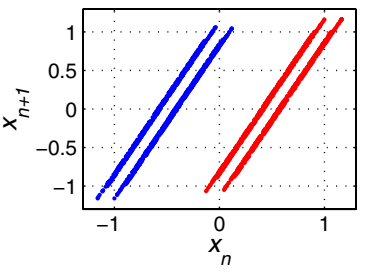

(b)

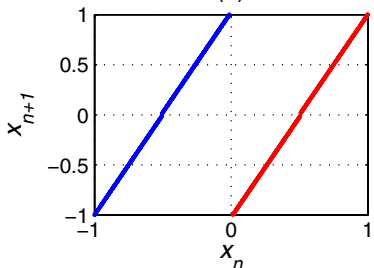

(d)

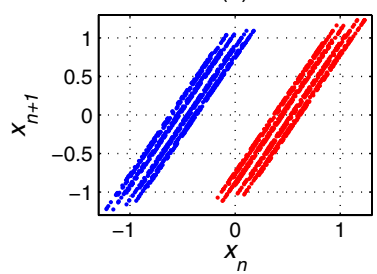

FIG. 1 (color online). (a) 3D reconstructed attractor using $x(t)$ in Eq. (7). (b) Poincaré map of $x(t)$ in Eq. (7). (c) Poincaré map of the signal received after being transmitted over a channel with two propagation paths and (d) over a channel with three propagation paths.

$\beta=0.9$, filter parameters $\alpha_{1}=\alpha_{2}=40$, and $\tau_{1}=0$, $\tau_{2}=2$, the sampled points of the received signal are used to construct the return map shown in Fig. 1(c). The return map of the signal received after being transmitted over a channel with three propagation paths with $\tau_{1}=0, \tau_{2}=2, \tau_{3}=3$ is shown in Fig. 1(d). From Figs. 1(c) and 1(d), one notices that all branches of the received signal have the same slope as the return map of the transmitted signal, meaning that the positive LE of the received signal equals the positive LE of the transmitted signal. To show that the slopes are indeed identical, notice that $x_{R}(n)=\sum_{j=1}^{L} w\left(n-\tau_{j}\right) e^{\beta \tau_{j}}=$ $\sum_{j=1}^{L}\left[\alpha_{1}^{d} w\left(n-\tau_{j}-1\right)\right] e^{\beta \tau_{j}}+\sum_{j=1}^{L}\left[\alpha_{2}^{d} x\left(n-\tau_{j}-1\right)\right] e^{\beta \tau_{j}}$, and $\quad x_{R}(n+1)=\sum_{j=1}^{L}\left[\alpha_{1}^{d} w\left(n-\tau_{j}\right)\right] e^{\beta \tau_{j}}+\sum_{j=1}^{L}\left[\alpha_{2}^{d} x(n-\right.$ $\left.\left.\tau_{j}\right)\right] e^{\beta \tau_{j}}$; therefore, $x_{R}(n+1)=\sum_{j=1}^{L} \alpha_{1}^{d} w\left(n-\tau_{j}\right) e^{\beta \tau_{j}+}$ $J \sum_{j=1}^{L} \alpha_{2}^{d} x\left(n-\tau_{j}-1\right) e^{\beta \tau_{j}}=\sum_{j=1}^{L} \alpha_{1}^{d} w\left(n-\tau_{j}\right) e^{\beta \tau_{j}}+$ $J\left[x_{R}(n)-\sum_{j=1}^{L} \alpha_{1}^{d} w\left(n-\tau_{j}-1\right) e^{\beta \tau_{j}}\right]$. This takes us to Eq. (1).

Information to be transmitted can be binary encoded in the transmitted signal by using $x_{n+1} \leq x_{n}$ to represent " 1 " (in red) and $x_{n+1}>x_{n}$ to represent " 0 " (in blue) as shown in Fig. 1(b). Comparing Fig. 1(b) with Figs. 1(c) and 1(d), the same rules can be used to decode the information received. The different branches of the return map are created by the multipath of the channel. The separation between them depends on the damping coefficient. The number of branches observed in the return map for $x_{R}(n)$ depends on the number of branches of the $\left(1-\tau_{j}\right)$ order of the inverse linear map. Representing $w\left(n-\tau_{j}\right)$ by $J^{-\tau_{j}} w(n)$, we get

$$
x_{R}(n+1)=\sum_{j=1}^{L} J^{1-\tau_{j}} w(n) e^{\beta \tau_{j}} .
$$




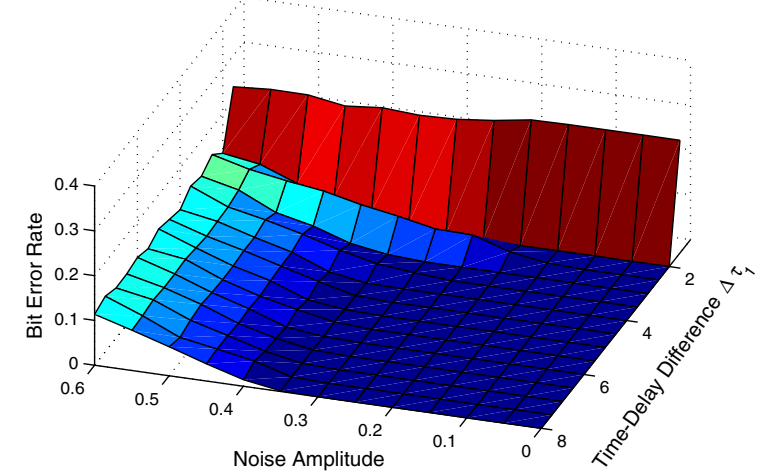

FIG. 2 (color online). Bit error rate for different time-delay differences, $\Delta \tau_{1}=\tau_{2}-\tau_{1}$, and the amplitude of the bounded noise.

Ignoring the damping and the filtering effects, if there are only two propagation paths, with $\tau_{1}=0$ and $\tau_{2}=2$, then $x_{R}(n+1)=J x(n)+J^{-1} x(n)$, meaning that in each sector of the map one should find two branches as seen in Fig. 1(c).

To analyze the performance of the proposed communication system, when there is noise in the channel, we consider the same parameters as in Fig. 1(b), but we now introduce a uniform bounded noise $\eta(t)$ in the CBWC. Results are shown in Fig. 2, which shows that information transmission happens reliably for large time-delay difference and large noise amplitude.

The information capacity of the CBWC, when the chaotic signal generated by Eq. (7) (or any other flow with one positive LE) is used, can be calculated as a function of the filter parameter $\alpha_{1}$. Since this oscillator has only one positive Lyapunov exponent, using the Lyapunov dimension formula, it yields the information capacity per iteration (corresponding to a time interval of $T_{s}=1$ ):

$$
\lambda_{1}<\left(D_{L}-2\right) \alpha_{1},
$$

where $D_{L}$ is the Lyapunov dimension.

If the transmitter is moving with respect to the receiver, the chaotic signal is altered due to the Doppler effect. The consequence is that its frequency changes as a function of the relative speed. This effect can be incorporated into the CBWC by rescaling the time derivative of the ODE system generating $x_{T}(t)$ and $w(t)$ [Eq. (3)] by $d \xi=\theta(\delta \dot{\mathbf{x}}, v) d t$, where $\delta \dot{\mathbf{x}}$ represents the relative speed between transmitter and receiver, $v$ is the speed of the signal propagation in the media, and $\theta(\delta \dot{\mathbf{x}}, v)$ represents the time transformation that models the Doppler effect in the signal $w(t)$, altering its instantaneous as well as its natural frequency. According to Ref. [40], if the time transformation $d \xi=\theta(\delta \dot{\mathbf{x}}, v) d t$ is applied to an ODE system, its dimension (a metric quantity) remains unaltered, but its LEs spectra $\lambda_{\mathrm{T}}$ (a time ratio quantity) is transformed according to $\lambda_{\mathbf{T}} /\langle\theta\rangle_{t}$. Therefore, if the Doppler effect is to be taken into consideration, the information capacity of our proposed communication system changes to

$$
\lambda_{1}<\langle\theta\rangle_{t}\left(D_{L}-2\right) \alpha_{1} .
$$

If transmitter and receiver are approaching (moving away) each other, $\langle\theta\rangle_{t}$ decreases (increases), as does the information capacity of the channel.

To summarize, we have shown analytically that the spectra of Lyapunov exponents of a received chaotic signal, after being transmitted through CBWC, contains the spectra of the Lyapunov exponents of the original transmitted signal. This physical property allows for the construction of a fully chaos-based wireless communication system. Such a communication system would have excellent performance when communicating in the presence of bounded noise. We calculate the information capacity of the CBWC and show that it is a function not only of the invariant properties of the chaotic signal, but also of the parameters of the channel and the relative motion between transmitter and receiver. Furthermore, if special chaotic signals are used, the channel capacity is unaffected by the presence of a multipath. This work provides a strong argument for using chaotic systems in communication.

This work is supported by NSFC-RSE Joint Project No. 443570/NNS/INT-61111130122, NSFC Grant No. 61172070, Scottish NRP, and the EPSRC Grant No. EP/I032606/1.

[1] T. S. Rappaport, Wireless Communications: Principles and Practice (Prentice-Hall, Englewood Cliffs, NJ, 1996).

[2] A. Goldsmith, Wireless Communications (Cambridge University Press, Cambridge, England, 2005).

[3] J. M. V. Grzybowski, M. Rafikov, and E.E. N. Macau, Acta Astronaut. 66, 1160 (2010).

[4] J. Heidemann, M. Stojanovic, and M. Zorzi, Phil. Trans. R. Soc. A 370, 158 (2011).

[5] M. Stojanovic and J. Preisig, IEEE Commun. Mag. 47, 84 (2009).

[6] Multipath propagation, http://en.wikipedia.org/wiki/ Multipath_propagation.

[7] J.G. Proakis and M. Salehi, Communication Systems Engineering (Prentice-Hall, Englewood Cliffs, NJ, 2002).

[8] M. L. Jakobsen, Ph.D. thesis, Aalborg University, 2009.

[9] S. Hayes, C. Grebogi, and E. Ott, Phys. Rev. Lett. 70, 3031 (1993).

[10] C. W. Wu and L. O. Chua, Int. J. Bifurcation Chaos Appl. Sci. Eng. 03, 1619 (1993).

[11] H. Dedieu, M. Kennedy, and M. Hasler, IEEE Trans. Circuits Syst. II 40, 634 (1993).

[12] K. M. Cuomo, A. V. Oppenheim, and S. H. Strogatz, IEEE Trans. Circuits Syst. II 40, 626 (1993).

[13] E. Bollt, Y.-C. Lai, and C. Grebogi, Phys. Rev. Lett. 79, 3787 (1997).

[14] E. Rosa, Jr., S. Hayes, and C. Grebogi, Phys. Rev. Lett. 78, 1247 (1997).

[15] M. Baptista, Phys. Lett. A 240, 50 (1998).

[16] G. Kolumbán, IEEE Trans. Circuits Syst. I 47, 1692 (2000). 
[17] M. S. Baptista and L. López, Phys. Rev. E 65, 055201 (2002).

[18] A. Abel and W. Schwarz, Proc. IEEE 90, 691 (2002).

[19] T. Yang, Int. J. Comput. Cogn. 2, 81 (2004).

[20] P. Stavroulakis, Chaos Applications in Telecommunication (Taylor \& Francis, London, 2006).

[21] S. Banerjee, M. Mitra, and L. Rondoni, Applications of Chaos and Nonlinear Dynamics in Engineering (Springer, New York, 2011).

[22] A. Argyris, D. Syvridis, L. Larger, V. Annovazzi-Lodi, P. Colet, I. Fischer, J. García-Ojalvo, C.R. Mirasso, L. Pesquera, and K. Alan Shore, Nature (London) 438, 343 (2005).

[23] R. Badii, G. Broggi, B. Derighetti, M. Ravani, S. Ciliberto, A. Politi, and M. A. Rubio, Phys. Rev. Lett. 60, 979 (1988).

[24] L. Q. Zhu, Y.-C. Lai, F. C. Hoppersteadt, and E. M. Bollt, Chaos 13, 410 (2003).

[25] H.-P. Ren, M.S. Baptista, and C. Grebogi, Int. J. Bifurcation Chaos Appl. Sci. Eng. 22, 1250199 (2012).

[26] M. Eisencraft, R. Attux, and R. Suyama, Chaotic Signals in Digital Communications (CRC Press, Boca Raton, FL, 2013).

[27] M. Eisencraft, R. Fanganiello, J. Grzybowski, D. Soriano, R. Attux, A. Batista, E. Macau, L. Monteiro, J. Romano, R. Suyama et al., Commun. Nonlinear Sci. Numer. Simul. 17, 4707 (2012).

[28] So far, few works have considered the chaos-based communication in a channel with multipath propagation $[29,30]$. But they use chaotic maps as a pseudorandom number generator to spread the spectrum. The signal transmitted in the channel is not chaotic. If a chaotic signal is transmitted in a wireless communication channel, the modification made to the properties and the information content of the chaotic signal becomes a rather fundamental problem. It has not been fully considered and assessed until now.

[29] M. Kennedy, G. Kolumban, G. Kis, and Z. Jako, IEEE Trans. Circuits Syst. I 47, 1702 (2000).

[30] G. Kaddoum, D. Roviras, P. Charge, and D. FournierPrunarety, in Proceeding of the IEEE International Symposium on Circuits and Systems (IEEE, Piscataway, NJ, 2009), pp. 2637-2640.

[31] D. Tse and P. Viswanath, Fundamentals of Wireless Communication (Cambridge University Press, Cambridge, England, 2005).

[32] See Supplemental Material at http://link.aps.org/ supplemental/10.1103/PhysRevLett.110.184101 for an illustration about how much a chaotic signal can be modified by the action of the multipath propagation and damping in the wireless channel, and the demonstration that the Lyapunov exponents are preserved in discrete chaotic signals transmitted over a wireless channel.

[33] K. Ogata, Modern Control Engineering (Prentice-Hall, Englewood Cliffs, NJ, 2010), 5th ed.

[34] E. N. Lorenz, J. Atmos. Sci. 20, 130 (1963).

[35] We also consider filters of higher order, such as a second order obtained by $\ddot{w}(t)=-\alpha_{1} \dot{w}(t)-\alpha_{2} w(t)+\alpha_{3} x_{T}(t)$, yielding a similar Jacobian matrix for the autonomous ODE system describing the dynamics of the wireless channel. In this case, the eigenvalues generated by the higher order filter appears in the diagonal of the Jacobian matrix, and, hence additional negative Lyapunov exponents corresponding to the (stable) filter are generated. For the channel containing more than two path propagations, we obtain a similar upper-triangular Jacobian matrix as Eq. (6), which only produces, in addition to the LEs of the original system and the filter, more negative infinite LEs due to more paths considered.

[36] In the general case, the practical communication channels are modeled by the linear filters (that only gives phase shift to the signal) $[1,2,7]$. If a physical media behaves as a nonlinear filter, it could be modeled by replacing the linear filters, Eq. (3), by a nonlinear system perturbed by the information signal $x_{T}(t)$. Denote this system by $\tilde{w}(t)$. Assuming that the damping can be separated from the nonlinear action of the filtering as in Eq. (2), this nonlinear filtered multipath channel could be modeled by $x_{R}(t)=$ $\sum_{j=1}^{L} e^{\beta \tau_{j}} \tilde{w}\left(t-\tau_{j}\right)$. The LEs of $\tilde{w}(t)$ are the ones that are now preserved. Ideally, these LEs could be identical to the ones from $x_{T}(t)$, if the action of the nonlinear filter behaves as a passive channel of communication [37] that only contributes with negative LEs and does not alter the information of the perturbing signal $x_{T}(t)$. Decoding the signal $x_{R}(t)$ out of $\tilde{w}$, in a general nonlinear filter situation, would be a remarkable task, but is out of the scope of this work. However, a simple pathway to solve this problem is by creating a signal $x_{T}(t)$ whose time scale is much smaller than the time scale of the nonlinear filter $\tilde{w}(t)$. That would result in a signal $\tilde{w}(t)$ that is the result of a modulation from $x_{T}(t)$. Consequently, decoding of $x_{R}(t)$ could be done by extracting the modulatory signal out of $\tilde{w}(t)$, for example, by using a classical approach of measuring the mean field.

[37] M. S. Baptista and J. Kurths, Phys. Rev. E 77, 026205 (2008).

[38] N. J. Corron, J.N. Blakely, and M. T. Stahl, Chaos 20, 023123 (2010).

[39] T. Matsumoto, Proc. IEEE 75, 1033 (1987).

[40] A. E. Motter, Phys. Rev. Lett. 91, 231101 (2003). 